\title{
William Campbell Little, M.D. In Memoriam
}

\author{
Ares Pasipoularides ${ }^{1}$
}

Received: 14 July 2015 / Accepted: 14 July 2015 /Published online: 5 August 2015

(C) Springer Science+Business Media New York 2015

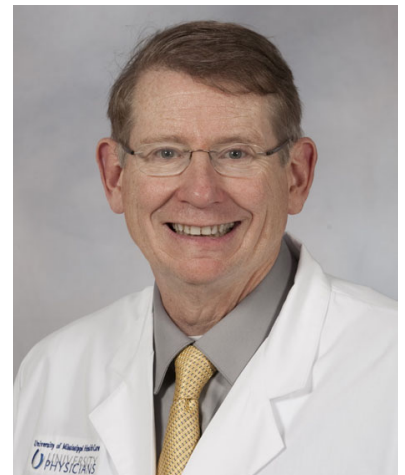

In the afternoon of July 9, 2015, Dr. William C. Little, a nationally recognized interventional cardiologist with research interests in cardiac function, experienced chest discomfort during a meeting at his home institution in Jackson Mississippi and died suddenly. He is survived by his wife Connie, his two children Elizabeth and John, and three grandchildren. Since August 2013, he was Professor and Chair of the Department of Medicine at the University of Mississippi Medical Center, overseeing a massive department with a broad range of faculty encompassing both general internists and specialists in hypertension, heart disease, lung disorders, immunology, digestive disorders, cancer treatment, geriatrics, and infectious diseases.

Dr. Little was born and raised in Ohio, the son of Dr. Robert C. Little, scientist, physician, and educator, of whom he was

Editor-in-Chief Jennifer L. Hall oversaw the review of this article

Ares Pasipoularides

apasipou@duke.edu

1 Duke University School of Medicine and Graduate School, Durham, NC, USA very proud and whose textbook, "The Physiology of the Heart and Circulation," now in its 4th Edition, has been widely used by medical students in this country and abroad. After graduating from Oberlin College and earning his M.D. from the Ohio State University College of Medicine, Bill Little completed his internal medicine residency at the University of Virginia Hospitals, followed by a cardiology fellowship at the University of Alabama at Birmingham. He held faculty positions at UAB and at the University of Texas Health Science Center in San Antonio. In 1986, he moved to Wake Forest University Baptist Medical Center in Winston-Salem, N.C., where he served as the McMichael Professor and Chief of Cardiology, Vice Chair of the Department of Internal Medicine and on the Board of Trustees of North Carolina Baptist Hospital. He had been in practice for over 40 years. He served as the Chair of the Cardiovascular Disease Board and as member of the Board of Directors of the American Board of Internal Medicine and participated on the steering committees of several international clinical trials. The ABIM certifies physicians in internal medicine, as well as 19 subspecialties, including cardiovascular disease.

An accomplished researcher, Dr. Little was the author of numerous influential scientific publications. His investigations have improved the understanding of the causes of heart attacks, of how blood fills both normal and diseased hearts and of the use of pacemakers to treat heart failure. He always strived to see basic science knowledge translated and moved into clinical practice. His contributions have been recognized by election to the American Society of Clinical Investigation and the Association of American Physicians, as well as the Lamport Award for Cardiovascular Research from the American Physiological Society, the Harrison Award of the Southern Society for Clinical Investigation, and the 2010 Laennec Master Clinician Award of the American Heart Association.

He will be greatly missed. 